\title{
LINK OF THE HIPPARCOS CATALOGUE TO THE ICRS
}

\author{
J. KOVALEVSKY \\ $O C A / C E R G A$ \\ UMR CNRS 6527 \\ Av. Copernic, 06130-Grasse (France)
}

\begin{abstract}
In compliance with the 1991 IAU resolutions on reference frames, the merged Hipparcos Catalogue had to be aligned in such a way that it represents at optical wavelengths the International Celestial Reference System (ICRS). This was realized as a combination of particular solutions obtained from observations of radio stars by radio interferometry, observation of quasars relative to Hipparcos stars, photographic plates including galaxies and Hipparcos stars, star catalogues referred to extragalactic objects, HST observations, and comparison of Earth orientation parameters obtained by VLBI and ground-based optical observations reduced with Hipparcos positions and proper motions. The individual results generally agree within 10 mas in orientation at epoch and $1 \mathrm{mas} / \mathrm{yr}$ in spin of the system. Two methods were used to combine the results and yielded uncertainties of $0.6 \mathrm{mas}$ in orientation and $0.25 \mathrm{mas} / \mathrm{yr}$ in spin.
\end{abstract}

\section{Introduction}

A set of recommendations within IAU resolution A4 of 1991 (Bergeron, 1992) on celestial reference systems and frames called for the definition of a celestial reference system that would have no global rotation with respect to a set of distant extragalactic objects. This system, called the International Celestial Reference System (ICRS, see Arias et al., 1995) was recognized by the IAU Working Group on Reference Frames and materialized in the form of a catalogue of positions of 608 objects (Ma and Feissel, 1997) adopted by the IAU as the International Celestial Reference Frame (ICRF) during this General Assembly. This catalogue was prepared in time to be available when the merged Hipparcos Catalogue was completed as a combination of the two catalogues prepared by the two data reduction consortia (FAST, see Kovalevsky et al., 1992 and NDAC, see Lindegren et al., 1992). The merging procedure is described by Arenou (1997) and the provisional astrometric parameters obtained were made available to all participants of the preparation of the link. The ensemble of the work done in this respect is described in Kovalevsky et al., 1997a.

\section{Methods and observations}

Let the extragalactic reference frame ICRF be represented by the triad of unit vectors $E=\left(\mathbf{x}_{E}, \mathbf{y}_{E}, \mathbf{z}_{E}\right)$ and the Hipparcos provisional reference frame by $H=\left(\mathbf{x}_{H}, \mathbf{y}_{H}, \mathbf{z}_{H}\right)$. The rclation between them can be represented by a time dependent vector

$$
\mathbf{V}(t)=\varepsilon+\left(t-t_{0}\right) \omega
$$

where $\varepsilon$ is the rotation vector at the mean epoch of the Hipparcos Catalogue, $t_{o}=1991.25$ and called here orientation. While $\omega$ is a vector representing the yearly variation, called here spin.

The principle of the determination of either of these vectors was first given by Froeschlé \& Kovalevsky (1982) for most of the possible cases. It was applied on eleven different sets of data: 
1. VLBI observations of 12 radio-emitting stars by several networks (Lestrade et al., 1995) provided both $\varepsilon$ and $\omega$ with uncertainties of the order of 0.5 mas for the components of $\varepsilon$ and $0.3 \mathrm{mas} / \mathrm{yr}$ for those of $\omega$.

2. Connected radio interferometer MERLIN (Morrison et al., 1997) observed 13 radio stars, but with insufficient spread in time to get reliable results in $\omega$. So, only the components of $\varepsilon$ were given within 2.6 mas standard error.

3. VLA observations, mostly done around 1983-86 (Florkowski et al., 1985) and with some additional data in 1994-95, gave standard uncertainties of the order of 5 mas on the components of $\varepsilon$.

4. Optical positions of compact sources derived from CCD observations of 78 selected sources (Johnson et al., 1995 and Zacharias et al., 1995) gave an overall precision of the same order of magnitude as 3 above.

5. About 80 observations of separations of 46 Hipparcos stars to extragalactic objects were made on the Hubble Space Telescope. These observations were insufficient in number, so that the uncertainties ( 5 mas in $\varepsilon$ and 2 mas/yr in $\omega$ ) were rather disappointing.

6. The Lick proper motion survey referred to galaxies allowed the determination of $\omega$. Two solutions were obtained respectively by I. Platais and his co-workers and S. Röser. The results for $\omega$ were scattered, apparently due to strong magnitude errors.

7. A similar work on the results of the Yale-San Juan Southern Proper Motion Program led to values that were about $0.5 \mathrm{mas} / \mathrm{yr}$ from the mean (Platais et al., 1997), a very satisfactory result, even if the internal errors have been very underestimated.

8. A similar overall precision on $\omega$ was obtained with the catalogue of faint stars KSZ (Kislyuk et al., 1997) using 415 stars from 154 areas on the sky.

9. A special programme of determination of proper motions from widely separated in time photographic plates (70 to 100 years) using 88 Hipparcos stars in 13 fields (Tucholke et al., 1997) yielded actual uncertainties of the order of $0.5 \mathrm{mas} / \mathrm{yr}$ in $\omega$.

10. A similar programme based on 24 fields taken at the Tautenburg Schmidt Telescope with time intervals of 20 to 40 years gave better results and better uncertainties (Hirte et al., 1997).

11. Finally, a comparison was made between the Earth rotation parameters determined with VLBI in the ICRS system and those obtained from optical observations rediscussed with Hipparcos data. Many uncertainties are present in this method that could not be evaluated and whilst the results obtained are rather good in $x$ and $y$ components of $\omega$, the results in $\varepsilon$ were not satisfactory (Vondrák et al., 1997).

\section{Combination of results}

The eight results for $\omega$ and six results for $\varepsilon$ were discussed as described in Lindegren and Kovalevsky (1995) and in Kovalevsky et al., 1997a. Two different methods were used, either separating $\varepsilon$ and $\omega$ which are only slightly correlated, or in treating all unknowns together. Actually, both methods differed also slightly in the weights attributed to each result.

The solutions obtained in $\varepsilon$ differed by less than 0.05 mas in $x$ and $z$ components and 0.16 in the $y$ component. Similarly, the solutions for $\omega$ differed by less than 0.08 mas $/ \mathrm{yr}$ in $x$ and $z$ and $0.13 \mathrm{mas} / \mathrm{yr}$ in $y$. The weighted standard errors were also quite similar. This led to the adoption of values for $\varepsilon$ and $\omega$ with respectively standard uncertainties of \pm 0.60 mas in all components of $\varepsilon$ and $\pm 0.25 \mathrm{mas} / \mathrm{yr}$ in all the three components of $\omega$. These values are to be compared with \pm 0.50 mas and $\pm 0.15 \mathrm{mas} / \mathrm{yr}$ as mean values obtained by both solutions for the standard errors. So, a rather large security factor has been retained. In this manner, the interval of confidence includes the spread of values obtained by various solutions with different choices of weights.

In addition, an external check was obtained using a statistical model of stellar motion (Kovalevsky et al., 1997b) for a determination of the components of $\omega$ in the galactic plane. The results obtained depend on distance, galactic latitude and colour, but they are all compatible within their own errors with the uncertainty in $\omega$ stated above.

So, in conclusion, the rotation components that resulted from this work and that were applied to the preliminary merged catalogue to obtain the final Hipparcos Catalogue and the associated uncertainties appear to be consistent and represent what was achievable. 


\section{References}

Arenou, F., 1997, The Hipparcos and Tycho Catalogues, ESA Publ. SP-1200, 3, 369-385

Arias, E.F., Charlot, P., Feissel, M. and Lestrade, J-F., 1995, A\&A, 303, 604-608

Bergeron, J., 1992, Proceedings of the twenty first General Assembly of the IAU, Kluwer Acad. Publ., Dordrecht, 41-64.

Froeschlé, M. and Kovalevsky, J., 1982, A\&A, 116, 89-96

Hirte, S., Schilbach, E. and Scholz, R-D., 1997, A\&A Suppl., in press

Johnston, K.J., Fey, A.L., Zacharias, N. et al., 1995, Astron. J., 110, 880-915

Kislyuk, V.S., Rybka, S.P., Yatsenko, A.I. and Kharchenko, N.V., 1997, A\&A, 321, 660-664

Kovalevsky, J., Falin, J-L., Pieplu, J-L. et al., 1992, A\&A, 258, 7-17

Kovalevsky, J., Lindegren, L., Perryman, M.A.C. et al., 1997a, A\&A, 323, 620-633

Kovalevsky, J., Lindegren, L. and Perryman, M.A.C., 1997b, The Hipparcos and Tycho Catalogues, ESA Publ. SP$1200,3,410$ and foll.

Lestrade, J-L., Jones, D.L., Preston, R.A. et al., 1995, A\&A, 304, 182-188

Lindegren, L., Hoeg, E, van Leeuwen, F. et al., 1992, A\&A, 258, 18-30

Lindegren, L. and Kovalevsky, J., 1995, A\&A, 304, 189-201

Ma, C. and Feissel, M., 1997, IERS Technical Note 23, Observatoire de Paris

Morrison, L.V., Garrington, S.T., Argyle, R.W. and Davis, R.J., 1997, Hipparcos Symposium, Venice, ESA Publ. SP-402, 143-146

Platais, I., Kozhurina-Platais, V., Girard, T.M. et al, 1997, Hipparcos Symposium, Venice, ESA Publ. SP-402, 153-156

Tucholke, H-J., Brosche, P. and Odenkirchen, M., 1997, A\&A Suppl., 122, 433-437

Vondrák, J., Ron, C. and Pešek, I., 1997, A\&A, 319, 1020-1024

Zacharias, N., de Vegt, C., Winter, L. and Johnston, K.J., 1995, Astron. J., 110, 3093-3106 